Rheological stability with couple stresses and its application to geological folding

\author{
Bу M. A. Biot
}




\title{
Rheological stability with couple stresses and its application to geological folding*
}

\author{
Bх М. А. Віот \\ 300 Central Park West, New York, N.Y., U.S.A. \\ (Communicated by G.Temple, F.R.S.--Received 14 July 1966)
}

\begin{abstract}
The author's theory of elasticity and viscoelasticity of initially stressed anisotropio solids is extended to include couple stresses and an additional refinement represented by a dependence of the deformation on the second gradient of the stress. The theory is intended to provide an approximate continuous model valid over a wide range for the mechanics of laminated media. The equations derived for the continuous nodel are rigorous for a medium initially at rest under the initial stress but are applicable as an approximation to stability problems of viscous and plastic media which exhibit initial strain rate with finite strain. The effect of gravity is included. A 'couple-stress analogy' renders immediately applicable to the medium with couple stresses a large class of solutions obtained earlier for plates and multilayers under initial stress. The problem of internal buckling is discussed. The theory is particularly suited to the analysis of folding and gravity instability of stratified sedimentary rock and remarkably simple results are shown to provide striking verification when applied to a wide variety of geological structures.
\end{abstract}

\section{INTRODUCTION}

The use of couple stresses or moments per unit area acting internally in an elastic continuum is found in implicit form in a paper by Cauchy $\dagger$ as long ago as 1851 . That paper represented a quite advanced form of the theory where the energy density is assumed to be a function of gradients of the displacement of any order. The next systematic development is due to E. \& F. Cosserat (I909) who introduced explicitly the couple stresses in the analysis of a system with microelements undergoing rotations which are different from the local rotation of the continuum. In recent years the subject has been treated extensively by several authors introducing a variety of stress-strain relations which involve couple stresses.

These theories deal with materials initially stress-free. In order to treat problems of stability for a medium with couple stresses we will generalize the theory to include the effect of initial stress.

The author has also discussed the effect of a new and additional feature by considering that the strain depends also on the second gradient of the stress. This effect was already introduced earlier in the context of geology (Biot 1964a, 1965 d). In the case of a laminated medium the effect leads to an 'interstitial flow correction' as outlined below at the end of $\S 8$.

Since we consider the very complex case of anisotropic elastic and viscoelastic media drastic simplification is achieved by following the direct procedure used in developing the mechanics of incremental deformations (Biot $1939,1965 a$ ) where the initial stress is rotated locally with the medium. The adjunction of couple stresses

* Work sponsored by the Shell Development Company, New York.

$\uparrow$ The existence of this paper was brought to the author's attention by R. D. Mindlin. 
into the theory is quite straightforward. The present treatment is intended to provide an approximate continuous and anisotropic model for the mechanics of rheological instability of laminated media. Such a theory is of importance in tectonic problems of geological folding as well as in many areas of technology. Only the geological applications will be discussed here.

The paper provides a unified treatment of many cases which the author has analysed previously by using suitable approximations for each separate problem. Complete solutions are obtained for the rheological behaviour under initial stress of extremely complex stratified media including the effect of gravity. A 'couple-stress analogy' results in the immediate applicability to a medium with couple stresses of a large class of results obtained earlier for plates and multilayers.

\section{Compressible media}

For the purpose of simplifying the algebra the theory is presented in the context of incompressibility. This does not affect the generality of the conclusions. Compressibility introduces mainly a correction. However, extension of the theory for this case is readily obtained by following exactly the procedure of this paper adding couple stresses to the author's general equations for the initially stressed compressible medium.

\section{ANISOTROPIO mLASTICITY WITH INITIAL STRESS}

In order to derive equations for the initially stressed medium with stress couples we first recall briefly the theory of elasticity of initially stressed anisotropic solids without stress couples (Biot 1939, 1963a, 1965a).

We shall limit ourselves to plane strain in the $x y$ plane. The material is assumed incompressible and orthotropic with directions of symmetry along $x$ and $y$. Initial principal stresses along these directions are $S_{11}$ and $S_{22}$. The displacements $u, v$ along $x$ and $y$ yield the incremental strain

$$
\begin{gathered}
e_{x x}=\frac{\partial u}{\partial x} \quad e_{y y}=\frac{\partial v}{\partial y} \quad e_{x y}=\frac{1}{2}\left(\frac{\partial v}{\partial x}+\frac{\partial u}{\partial y}\right) \\
\omega=\frac{1}{2}\left(\frac{\partial v}{\partial x}-\frac{\partial u}{\partial y}\right) .
\end{gathered}
$$

The incremental strain is associated with stress increments $s_{11}, s_{22}, s_{12}$ superposed upon the state of initial stress. These stress increments are referred to local axes which rotate with the material through an angle $\omega$. The incremental stress-strain relations are

$$
\left.\begin{array}{r}
s_{11}-s=2 N e_{x x}, \\
s_{22}-s=2 N e_{y y}, \\
s_{12}=2 Q e_{x y}
\end{array}\right\}
$$

where $N$ and $Q$ are elastic coefficients for incremental deformations. Since the medium is incompressible we write

$$
e_{x x}+e_{y y}=0
$$


This implies $s=\frac{1}{2}\left(s_{11}+s_{22}\right)$. The equilibrium conditions of the stress field are

$$
\left.\begin{array}{l}
\frac{\partial s_{11}}{\partial x}+\frac{\partial s_{12}}{\partial y}-P \frac{\partial \omega}{\partial y}=0 \\
\frac{\partial s_{12}}{\partial x}+\frac{\partial s_{22}}{\partial y}-P \frac{\partial \omega}{\partial x}=0
\end{array}\right\}
$$

with the initial stress difference $\quad P=S_{22}-S_{11}$.

If $S_{22}=0$ the quantity $P$ represents a compression acting in the $x$ direction.

\section{Alternative formulation}

The foregoing equations provide a rigorous formulation of the theory of elasticity in the presence of initial stress. For the particular purpose of this paper it is convenient to introduce an alternative formulation which provides an immediate intuitive interpretation.

We consider the alternative stress components

$$
\left.\begin{array}{rl}
t_{11} & =s_{11}-P e_{y y}, \\
t_{22} & =s_{22}, \\
t_{12}^{\prime} & =s_{12}+P e_{x y} .
\end{array}\right\}
$$

These quantities were introduced and discussed earlier (Biot $1939, \mathrm{I}_{963} a, b, \mathrm{I} 965 a$ ). In order to bring out their physical significance let us assume that $S_{22}=0$. Hence the initial stress is reduced to a compression $P=-S_{11}$ in the $x$ direction. In this case the components $t_{11}, t_{22}, t_{12}^{\prime}$ may be considered as the stresses per unit initial area referred to local axes which have rotated through the angle $\partial v / \partial x$. The physical significance of these stresses is illustrated in figure 1, showing the incremental stresses acting on a deformed element of the material. If the initial stress component $S_{22}$ is not zero it may be added as a uniform hydrostatic stress throughout. Since the medium is incompressible the deformation is not affected. The components $t_{11}$ and $t_{22}$ must then be interpreted as incremental forces in excess of those due to the hydrostatic stress and $P=S_{22}-S_{11}$ represents an 'effective' compressive stress.

The stress-strain relation may also be written differently. From equations $(2 \cdot 3)$, $(2 \cdot 4)$ and $(2 \cdot 7)$ we derive

$$
\left.\begin{array}{rl}
t_{11}-t_{22} & =4 M e_{x x} \\
t_{12}^{\prime} & =2 L e_{x y}
\end{array}\right\}
$$

with the coefficients

$$
\left.\begin{array}{rl}
M & =N+\frac{1}{4} P \\
L & =Q+\frac{1}{2} P .
\end{array}\right\}
$$

These coefficients were introduced and discussed earlier (Biot $1963 a, b, 1965 a$ ). They have a simple physical interpretation. We have referred to $L$ as the 'slide modulus' which relates the shear strain $e_{x y}$ to a tangential stress applied along a face parallel to the initial stress $P$ as shown in figure $1(b)$. The significance of the coefficient $M$ is obtained by considering the case $t_{22}=0$. The first of equations $(2 \cdot 8)$ yields

$$
t_{11}=4 M e_{x x} \text {. }
$$


Hence $4 M$ is the elastic coefficient relating the stress increment $t_{11}$ to the corresponding strain when no transverse stress $t_{22}$ is applied. Note that we may also put $t_{11}=0$. Since $e_{x x}=-e_{y y}$ we write $(2 \cdot 8)$ as

$$
t_{22}=4 M e_{y y}
$$

Hence either one of the two equations $(2 \cdot 10)$ or $(2 \cdot 11)$ may be used to define physically the coefficient $M$.

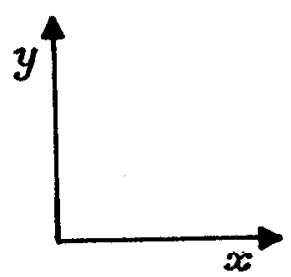

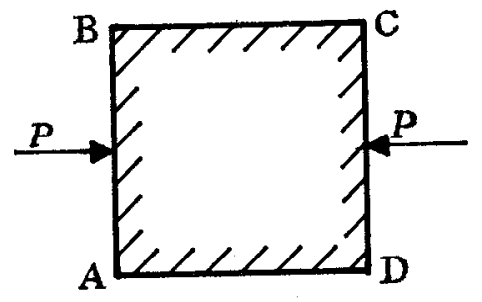

(a)

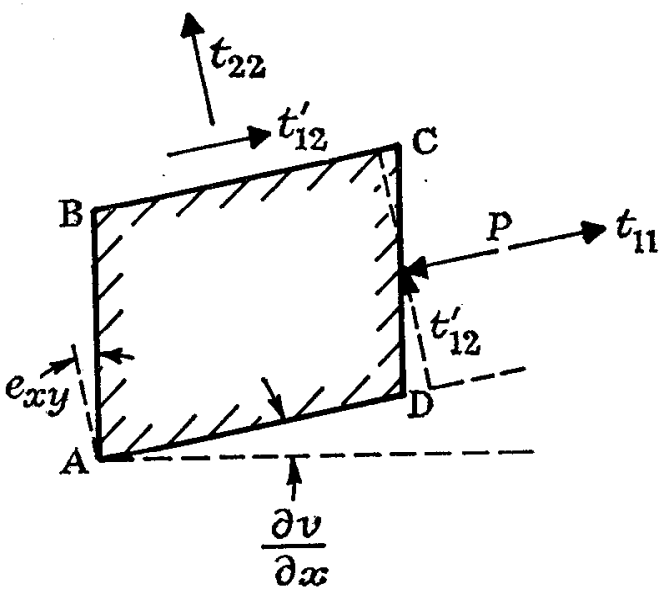

(b)

Figure 1. (a) Undeformed element of unit size under the initial stress $P$. (b) Deformed element, where $t_{12}^{\prime}$ and $t_{22}$ are the tangential and normal stresses on the face BC. The total force acting on the face $\mathrm{CD}$ in the direction $\mathrm{AD}$ is $t_{11}-P$ and the corresponding incremental stress per unit initial area is $t_{11}$.

Alternative equilibrium equations may also be written by solving equations $(2 \cdot 7)$ for $s_{11}, s_{22}, s_{12}$, and substituting these values in equations $(2 \cdot 5)$. We find

$$
\left.\begin{array}{l}
\frac{\partial t_{11}}{\partial x}+\frac{\partial t_{12}^{\prime}}{\partial y}=0, \\
\frac{\partial t_{12}^{\prime}}{\partial x}+\frac{\partial t_{22}}{\partial y}=P \frac{\partial^{2} v}{\partial x^{2}} .
\end{array}\right\}
$$

This form of the equation provides an immediate physical interpretation illustrated in figure 2. Consider a thin fibre of the material initially straight with its axis along the direction of $P$. After deformation the fibre acquires a curvature $\partial^{2} v / \partial x^{2}$ and the equilibrium condition of an element of this fibre is expressed by equations $(2 \cdot 12)$. 


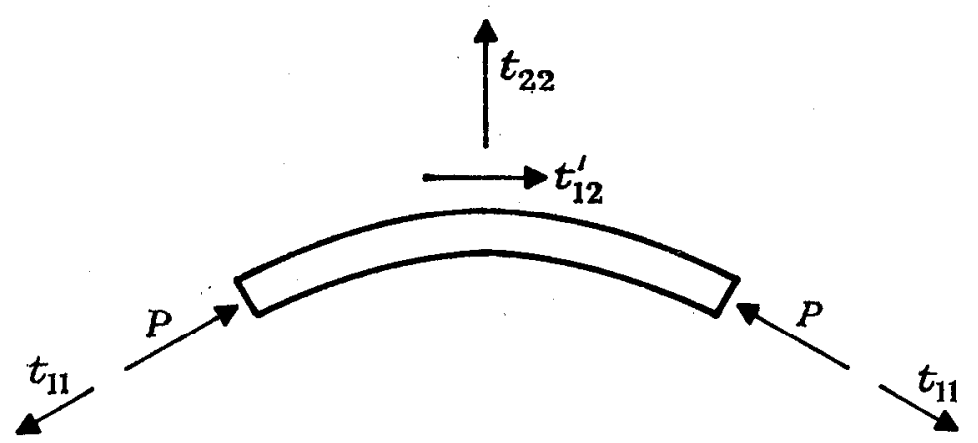

Figure 2. Physical interpretation of the equilibrium equations (2.12).

\section{Adjunction of COUPLE STRESSES}

We shall now examine the case where the medium exhibits a bending rigidity. Because of anisotropy the effect may be restricted to a couple $\mathscr{M}$ per unit arca acting on a surface initially perpendicular to the $x$ direction (figure 3 ). The value of

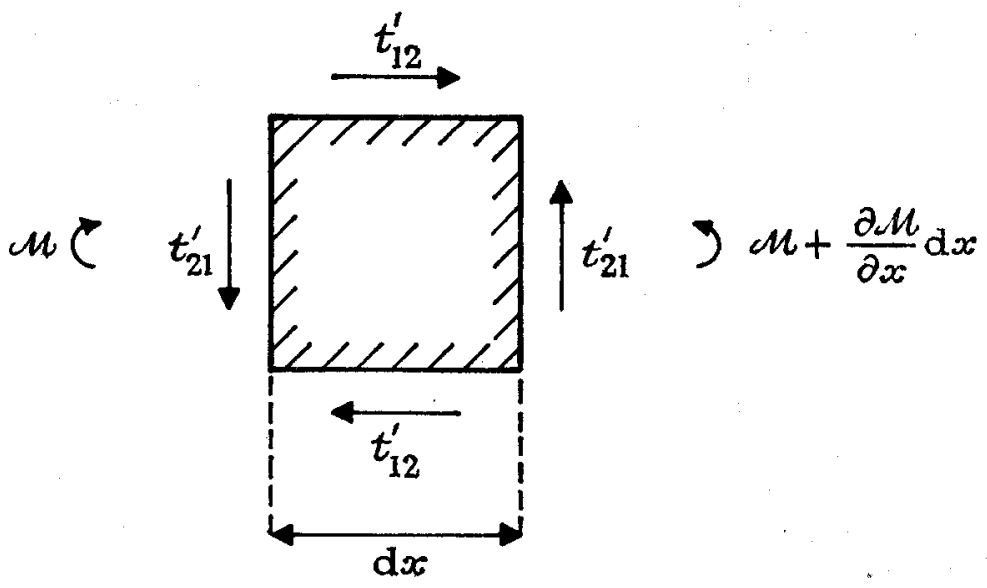

Fravre 3. Physical interpretation of the equilibrium equation $(3 \cdot 2)$ for moments.

this stress couple is assumed to be proportional to the curvature acquired by fibres of the medium initially parallel to the $x$ direction. Hence we may write

$$
\mathscr{M}=b \frac{\partial^{2} v}{\partial x^{2}} \text {. }
$$

The couple-stress coefficient $b$ is a measure of the 'bending rigidity' of the medium.

The presence of couple stresses implies nonsymmetric shear stress components $t_{12}^{\prime} \neq t_{21}^{\prime}$ as illustrated in figure 3 . Equilibrium of an element of the medium about an axis perpendicular to the figure implies the moment equation

$$
t_{12}^{\prime}-t_{21}^{\prime}=\frac{\partial \mathscr{M}}{\partial x} \text {. }
$$


Equilibrium in the vertical direction is expressed by the condition

$$
\frac{\partial t_{21}^{\prime}}{\partial x}+\frac{\partial t_{22}}{\partial y}=P \frac{\partial^{2} v}{\partial x^{2}}
$$

Hence the equilibrium equations $(2 \cdot 12)$ are now replaced by

$$
\left.\begin{array}{l}
\frac{\partial t_{11}}{\partial x}+\frac{\partial t_{12}^{\prime}}{\partial y}=0, \\
\frac{\partial t_{12}^{\prime}}{\partial x}+\frac{\partial t_{22}}{\partial y}=P \frac{\partial^{2} v}{\partial x^{2}}+\frac{\partial^{2} \mathscr{M}}{\partial x^{2}} .
\end{array}\right\}
$$

The second equation is obtained by eliminating $t_{21}^{\prime}$ between relations $(3 \cdot 2),(3 \cdot 3)$. The stress-strain relations $(2 \cdot 8)$ are not affected by the couple stresses and remain valid.

Equations (3.4) along with the stress-strain relations $(2 \cdot 8)$ the couple-stress relation $(3 \cdot 1)$ and the incompressibility condition $(2 \cdot 4)$ constitute a complete set which governs the deformation field.

\section{Variational principle}

The variational principles developed for the elastic continuum under initial stress (Biot 1939, I963a, 1965a) are readily extended to a medium with couple stresses. The energy density in this case becomes

$$
\Delta V=2 M e_{x x}^{2}+2 L e_{x y}^{2}+\frac{1}{2} b\left(\frac{\partial^{2} v}{\partial x^{2}}\right)^{2}-\frac{1}{2} P\left(\frac{\partial v}{\partial x}\right)^{2}
$$

For $b=0$ this expression becomes identical to results derived earlier.

\section{Couple-STress analogy and general solutions}

Let us assume that the deformation field is a sinusoidal function of $x$. In particular in this case the displacement may be written

$$
v=V(l y) \cos l x .
$$

Hence with the value $(3 \cdot 1)$ for the couple stress we may also write

$$
\frac{\partial^{2} \mathscr{M}}{\partial x^{2}}=-b l^{2} \frac{\partial^{2} v}{\partial x^{2}} \text {. }
$$

With this expression the equilibrium equations $(3 \cdot 4)$ become

$$
\left.\begin{array}{l}
\frac{\partial t_{11}}{\partial x}+\frac{\partial t_{12}^{\prime}}{\partial y}=0, \\
\frac{\partial t_{12}^{\prime}}{\partial x}+\frac{\partial t_{22}}{\partial y}=P^{\prime} \frac{\partial^{2} v}{\partial x^{2}}
\end{array}\right\}
$$

with

$$
P^{\prime}=P-b l^{2} \text {. }
$$

Equations $(4 \cdot 3)$ are identical to equations $(2 \cdot 12)$ provided $P$ is replaced by $P^{\prime}$. Hence for sinusoidal solutions of the type $(4 \cdot 1)$ the effect of stress couples is immediately 
derived by substituting for the actual compressive stress an analogue compressive stress into the general solutions of anisotropic elasticity with initial stress. We shall refer to this property as the couple-stress analogy.

General solutions for anisotropic elasticity with initial stress were derived in previous work (Biot $1963 a, b$, 1965a). They are immediately applicable to a medium with couple stresses provided we replace $P$ by $P^{\prime}$. These general solutions are written in the form

$$
\left.\begin{array}{rl}
u & =U(l y) \sin l x \\
v & =V(l y) \cos l x, \\
t_{11} & =r(l y) \cos l x \\
t_{22} & =q(l y) \cos l x, \\
t_{12}^{\prime} & =\tau(l y) \sin l x .
\end{array}\right\}
$$

In these expressions the factors function of $l y$ are obtained as follows

$$
\left.\begin{array}{rl}
l U & =-f^{\prime}, \\
l V & =f \\
r / L & =-f^{\prime}-f^{\prime \prime \prime} \\
q / L & =(2 m+1) f^{\prime}-f^{\prime \prime \prime}, \\
\tau / L & =-f^{\prime}-f^{\prime \prime}
\end{array}\right\}
$$

where $f$ is a function of $l y$ and the prime denotes a derivative of $f$ with respect to the argument $l y$. The function $f$ satisfies the ordinary fourth order differential equation,

and the coefficients are

$$
f^{\prime \prime \prime \prime}-2 m f^{\prime \prime}+k^{2} f=0,
$$

$$
\left.\begin{array}{c}
m=\frac{2 M-L}{L}, \\
k^{2}=\frac{L-P^{\prime}}{L}=\frac{L-P+b l^{2}}{L} .
\end{array}\right\}
$$

Note that the only place where the couple-stress coefficient appears is in the value of $k^{2}$. Solutions of the differential equation $(4 \cdot 7)$ are of the type $\exp (\beta l y)$ where $\beta$ satisfies the characteristic equation

$$
\beta^{4}-2 m \beta^{2}+k^{2}=0 .
$$

In particular we may write the general expression for $f$ in the form

$$
f=C_{1} \mathrm{e}^{\beta_{1} l y}+C_{2} \mathrm{e}^{\beta_{2} l y}+C_{3} \mathrm{e}^{-\beta_{1} l y}+C_{4} \mathrm{e}^{-\beta_{2} l y},
$$

where $C_{i}$ are four arbitrary constants. The four quantities $\pm \beta_{1}$ and $\pm \beta_{2}$ are the roots of the characteristic equation $(4 \cdot 9)$. Hence

$$
\left.\begin{array}{l}
\beta_{1}=\sqrt{ }\left\{m+\sqrt{ }\left(m^{2}-k^{2}\right)\right\} \\
\beta_{2}=\sqrt{ }\left\{m-\sqrt{ }\left(m^{2}-k^{2}\right)\right\} .
\end{array}\right\}
$$




\section{INTERNAL BUCKLING AND SHEAR THRESHOLD}

Consider the case

hence

$$
\begin{gathered}
2 M>L, \quad P^{\prime}>L \\
m>0, \quad k^{2}<0 .
\end{gathered}
$$

In the absence of couple stresses this corresponds to what the author has referred to as internal buckling of the first kind (Biot $1963 a, 1965 a$ ). One of the roots $\beta_{2}$ is a pure imaginary

$$
\beta_{2}=\mathrm{i} \xi \text {. }
$$

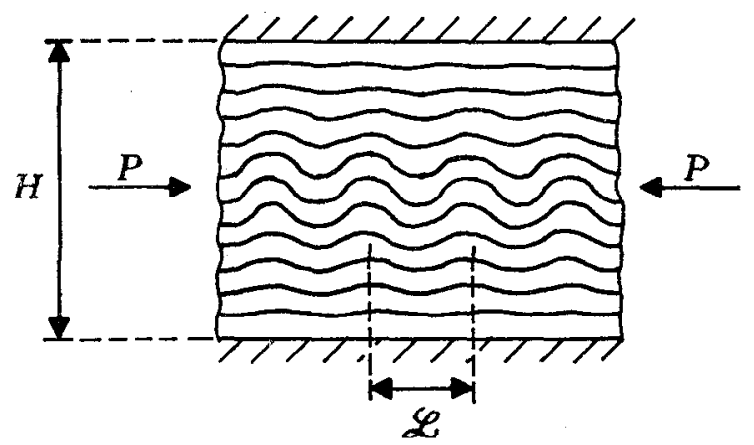

Figure 4. Pattern of internal buckling.

A particular solution $f$ is therefore

Let us put

$$
f=C \cos \xi l y .
$$

It is readily verified from equations $(4 \cdot 6)$ that the values of $V$ and $\tau$ vanish for $y= \pm \frac{1}{2} H$.

Hence the solution $(5 \cdot 4)$ represents an internal buckling of a medium confined without friction between rigid walls separated by a distance $H$. The deformation pattern is illustrated in figure 4. The wavelength of this pattern along $x$ is

$$
\mathscr{L}=2 \pi / l=2 \xi H \text {. }
$$

This provides a geometrical interpretation of the parameter $\xi=\mathscr{L} / 2 H$ as the ratio of the wavelengths along $x$ and $y$.

The characteristic equation $(4 \cdot 9)$ must be satisfied by substituting the root $\beta=\beta_{2}=\mathrm{i} \xi$. Hence

$$
\xi^{4}+2 m \xi^{2}+k^{2}=0 .
$$

From equations $(4 \cdot 8)$ and $(5 \cdot 5)$ we derive

$$
k^{2}=1-\frac{P}{L}+\frac{a}{\xi^{2}}
$$

with

$$
a=\pi^{2} b / L H^{2} \text {. }
$$


Substitution of the value $(5 \cdot 8)$ for $k^{2}$ into equation (5·7) yields

$$
\frac{P}{L}=1+\frac{a}{\xi^{2}}+2 m \xi^{2}+\xi^{4} .
$$

The term $a / \xi^{2}$ is due to the couple stress and plays a crucial role. Because of the presence of this term the value of $P$ goes through a minimum as a function of the wavelength parameter $\xi$. This minimum corresponds to incipient internal buckling. It is convenient to introduce a variable

$$
z=\xi(2 m / a)^{\frac{1}{2}} \text {. }
$$

With this variable equation $(5 \cdot 10)$ becomes

$$
\frac{P}{L}=1+\sqrt{ }(2 m a)\left[z^{2}+\frac{1}{z^{2}}\right]+\frac{a}{2 m} z^{4}
$$

Equating to zero the $z$ derivative of the right side of (5-12) yields

$$
z^{4}=1-\frac{1}{m} \sqrt{ }\left(\frac{2 a}{m}\right) z^{6} .
$$

The buckling wavelength is $\quad \mathscr{L}=2 H(a / 2 m)^{\frac{1}{4}} z$,

where $z$ is the positive real root of equation (5.13).

In many applications $a / m$ is small and a good approximation is $z=1$. The buckling wavelength becomes

$$
\mathscr{L}=2 H(a / 2 m)^{\frac{1}{1}},
$$

and the buckling load $P$ is given by

$$
P / L=1+2 \sqrt{ }(2 m a) .
$$

The case $a=0, P=L$, represents the shear threshold (Biot ig64a).

\section{Stability of plates and multilayers with couple stresses}

In previous work the author has derived complete solutions for the mechanics of plates and multilayers under initial stress (Biot $196_{3} b, c, 196_{5} a$ ). These solutions are immediately extended to plates and multilayers with couple stresses by using the couple-stress analogy derived in $§ 4$. By this analogy all equations derived for the continuous anisotropic medium are applicable to a medium with couple stresses provided we replace $P$ by $P^{\prime}$. For example, consider an isolated plate of thickness $h$ which is deformed sinusoidally along its axis $x$. 'The displacement and incremental stress fields are of the form $(4 \cdot 5)$. Values of the tangential and normal displacement and stress amplitudes $U, V, \tau, q$ are indicated by the subscript one on the top face and by the subscript two on the bottom face. Equations relating the four displacements to the four stresses are expressed in very condensed form by introducing the quadratic form

$$
\begin{aligned}
I=\frac{1}{2} A\left(U_{1}^{2}+U_{2}^{2}\right)-D U_{1} U_{2}+ & \frac{1}{2} C\left(V_{1}^{2}+V_{2}^{2}\right)+F V_{1} V_{2} \\
& +B\left(U_{1} V_{1}-U_{2} V_{2}\right)+E\left(U_{1} V_{2}-U_{2} V_{1}\right)
\end{aligned}
$$


with the six basic coefficients defined as follows:

$$
\left.\begin{array}{ll}
A=\frac{1}{2}\left(a_{11}+b_{11}\right), & D=\frac{1}{2}\left(a_{11}-b_{11}\right), \\
B=\frac{1}{2}\left(a_{12}+b_{12}\right), & E=\frac{1}{2}\left(a_{12}-b_{12}\right), \\
C=\frac{1}{2}\left(a_{22}+b_{22}\right), & F=\frac{1}{2}\left(a_{22}-b_{22}\right)
\end{array}\right\}
$$

and

$$
\left.\begin{array}{l}
a_{11}=\frac{\beta_{1}^{2}-\beta_{2}^{2}}{z_{1}-z_{2},} \\
a_{12}=\frac{\beta_{1}^{2} z_{1}-\beta_{2}^{2} z_{1}}{z_{1}-z_{2}}-1, \\
a_{22}=a_{11} z_{1} z_{2}, \\
b_{11}=\frac{\beta_{1}^{2}-\beta_{2}^{2}}{\beta_{1}^{2} z_{2}-\beta_{2}^{2} z_{1}} z_{1} z_{2}, \\
b_{12}=\beta_{1}^{2} \beta_{2}^{2} \frac{z_{1}-z_{2}}{\beta_{1}^{2} z_{2}-\beta_{2}^{2} z_{1}}-1, \\
b_{22}=b_{11} \frac{\beta_{1}^{2} \beta_{2}^{2}}{z_{1} z_{2}},
\end{array}\right\}
$$

where

$$
\left.\begin{array}{l}
z_{1}=\beta_{1} \tanh \left(\frac{1}{2} \beta_{1} l h\right), \\
z_{2}=\beta_{2} \tanh \left(\frac{1}{2} \beta_{2} l h\right) .
\end{array}\right\}
$$

With these definitions the surface stresses are given in terms of the four displacements by the relations

$$
\left.\begin{array}{ll}
\tau_{1}=l L \frac{\partial I}{\partial U_{1}}, & \tau_{2}=-l L \frac{\partial I}{\partial U_{2}}, \\
q_{1}=l L \frac{\partial I}{\partial V_{1}}, & q_{2}=-l L \frac{\partial I}{\partial V_{2}} .
\end{array}\right\}
$$

These equations are formally the same as those derived for a medium without couple stresses. The effect of the couple stresses is obtained by replacing $P$ by $P^{\prime}=P-b l^{2}$ in the value of $k^{2}$ as in equations $(4 \cdot 8)$.

Consider for example the problem of buckling of a plate with couple stresses subject to an initial compression $P$ along its axis. The surface stresses vanish, hence $\tau_{1}=q_{1}=\tau_{2}=q_{2}=0$. The buckling condition is obtained by equating to zero the determinant of equations $(6 \cdot 5)$. Since the buckling deformation is antisymmetric $\left(U_{1}=-U_{2}, V_{1}=V_{2}\right)$ the determinant is a two-by-two determinant and the buckling condition becomes

$$
a_{11} a_{22}-a_{12}^{2}=0 \text {. }
$$

After elimination of the factor $\left(z_{1}-z_{2}\right)$ it is written

$$
\left(\beta_{1}^{2}+1\right)^{2} z_{2}-\left(\beta_{2}^{2}+1\right)^{2} z_{1}=0
$$

For a given value of the parameter $M / L$ we may solve equation (6.7) for $P^{\prime} \mid L$ as a function of $l h=2 \pi h / \mathscr{L}$, where $\mathscr{L}$ is the buckling wavelength. This plot is illustrated schematically by the dashed line in figure 5 . It represents the buckling load $P^{\prime}$ in the 
absence of couple stresses. The horizontal asymptote $P^{\prime} \mid L \cong 1$ corresponds to pure shear or surface buckling while the parabolic portion near the origin corresponds to the Euler buckling at large wavelength. The actual buckling load $P$ in the presence of stress couples is obtained by plotting

$$
\frac{P}{L}=\frac{P^{\prime}}{L}+\frac{b t^{2}}{L}
$$

which is represented by the full line in figure 5. Since $b l^{2} / L$ may be written $\left(b / L h^{2}\right) l^{2} h^{2}$ we see that there is a double family of curves with two parameters $M / L$ and $b / L h^{2}$. The first parameter represents the anisotropy and the second the couple stress.

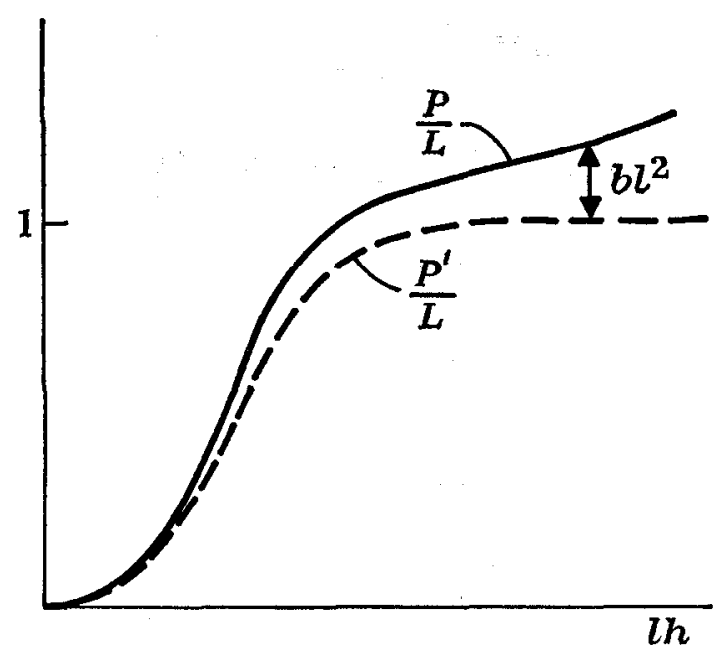

FIGURE 5. Critical buckling value of $P / L$ as a function of the wavelength parameter $l h=2 \pi h / \mathscr{L}$ for an anisotropic plate with couple stresses.

The analysis of a multilayered incompressible medium with couple stresses under initial stress is carried out in the same way. The system is constituted by superposed adhering horizontal plates of orthotropic symmetry along the horizontal and vertical directions. The principal initial stresses are oriented along the axes of symmetry. Elastic properties and initial stresses may be different in each layer. In the $i$ th layer of thickness $h_{i}$ the incremental elastic coefficients are $M_{i}$ and $L_{i}$, the effective compressive stress $P_{i}$, the couple stress coefficient $b_{i}$. The displacements at the top interface of the layer are designated by $U_{i} V_{i}$ and at the bottom interface by $U_{i+1}, V_{i+1}$.

In order to analyse this system we construct a quadratic form $I_{i}$ corresponding to the $i$ th layer. It is obtained from expression (6.1) by substituting $U_{i}, V_{i}, U_{i+1}, V_{i+1}$ for $U_{1}, V_{1}, U_{2}, V_{2}$ respectively. The coefficients $A, B, \ldots$, etc., are evaluated for the $i$ th layer as functions of $M_{i}, L_{i}, b_{i}, P_{i}$ and $l h_{i}$. The quadratic form for the whole multilayered system of $n$ layers is

$$
\mathscr{I}=\sum_{i=1}^{n} L_{i} I_{i}
$$


where the summation is extended to all layers. This quadratic form is a function of the $2 n+2$ boundary displacements of the layers. Buckling is governed by the system of $2 n+2$ homogeneous equations

$$
\frac{\partial \mathscr{I}}{\partial U_{i}}=0 \quad \frac{\partial \mathscr{I}}{\partial V_{i}}=0
$$

They are recurrence equations between the six interfacial displacements at three successive interfaces. The buckling condition is obtained by equating to zero the determinant of equations $(6 \cdot 10)$. Numerical programming procedures are discussed elsewhere (Biot $1963 c, 1965 a$ ).

Gravity may be taken into account by considering an analogue model derived earlier (Biot 1959, $1963 c, 1965 a$ ). In the model the effect of gravity is equivalent to applying vertical interfacial forces proportional to the change in altitude and the density difference. This amounts to using for $\mathscr{I}$ the expression

$$
\mathscr{I}=\sum_{i=0}^{n} L_{i} I_{i}+\mathscr{G}
$$

where

$$
\mathscr{G}=\frac{g}{2 l} \sum_{i=0}^{n}\left(\rho_{i+1}-\rho_{i}\right) V_{i+1}^{2}
$$

In this expression $g$ is the acceleration of gravity and is the mass density of the $i$ th layer. If the top surface of the structure is free we put $\rho_{0}=0$.

If the bottom layer adheres to a rigid surface we put $U_{n+1}=V_{n+1}=0$. Both or one of the end layers may be infinitely deep, corresponding to multilayers bounded by one or two half spaces. This case is included by putting equal to infinity the thickness of one or both of the end layers (numbered $i=0, i=n+1$ ).

\section{Couple StResses as an approximation for laminated media}

Consider a laminated medium composed of elastic layers of two different incompressible materials. A layer of thickness $h_{1}$ and elastic coefficients $M_{1}, L_{1}$ alternates with a layer of thickness $h_{2}$ and elastic coefficients $M_{2}, L_{2}$. We shall refer to them as 'layer 1 ' and 'layer 2 ' respectively. The layers are parallel to a plane of orthotropic symmetry of the materials. The principal initial stresses are oriented along the axes of orthotropic symmetry. The effective compressive initial stresses are $P_{1}$ for layer 1 and $P_{2}$ for layer 2.

It was shown (Biot $1963 a, 1965 a$ ) that for deformations of sufficiently large wavelength the medium behaves approximately as an anisotropic continuum with average incremental elastic coefficients $M, L$ and initial compressive stress $P$ equal to

$$
\left.\begin{array}{c}
M=M_{1} \alpha_{1}+M_{2} \alpha_{2}, \\
L=1 /\left(\frac{\alpha_{1}}{L_{1}}+\frac{\alpha_{2}}{L_{2}}\right), \\
P=P_{1} \alpha_{1}+P_{2} \alpha_{2},
\end{array}\right\}
$$

where

$$
\alpha_{1}=\frac{h_{1}}{h_{1}+h_{2}}, \quad \alpha_{2}=\frac{h_{2}}{h_{1}+h_{2}} \text {. }
$$


Hence the parameters $\alpha_{1}, \alpha_{2}$ represent the fraction of total thickness occupied by each layer.

The coefficients (7.1) may be said to represent the collective anisotropic behaviour of the laminated medium as contrasted with the intrinsic anisotropy of the layers themselves. For example, layers of isotropic materials with strong contrast of rigidity lead to collective coefficients $(7 \cdot 1)$ which correspond to a large collective anisotropy. For shorter wavelengths a correction must be introduced corresponding to the bending stiffness of the layers by considering an equivalent continuous medium with couple stresses. The couple-stress coefficient is evaluated as follows. Assume a deformation such that the displacement $v$ and the tangential stress $t_{12}^{\prime}$ are both independent of $y$. A closer examination of the problem shows that these assumptions are approximately valid in the range where the influence of couple stresses if significant. Consider the stress-strain relation

$$
t_{12}^{\prime}=L_{1}\left(\frac{\partial v}{\partial x}+\frac{\partial u}{\partial y}\right)
$$

in layer 1. Since $e_{x x}=\partial u / \partial x$ we may write

$$
\frac{\partial t_{12}^{\prime}}{\partial x}=L_{1}\left(\frac{\partial^{2} v}{\partial x^{2}}+\frac{\partial e_{x x}}{\partial y}\right)
$$

On the other hand

$$
t_{12}^{\prime}=L(\partial v / \partial x)
$$

where $L$ is the average slide modulus $(7 \cdot 1)$ for the laminated medium. Elimination of $t_{12}^{\prime}$ between equations $(7 \cdot 4)$ and $(7 \cdot 5)$ yields

$$
\frac{\partial e_{x x}}{\partial y}=\frac{\alpha_{2}\left(L_{2}-L_{1}\right)}{\alpha_{1} L_{2}+\alpha_{2} L_{1}} \frac{\partial^{2} v}{\partial x^{2}} \text {. }
$$

In deriving this expression we have substituted the value (7-1) for $L$ and used the identity $\alpha_{1}+\alpha_{2}=1$. Equation (7.6) shows that $e_{x x}$ is a linear function of $y$. Therefore $t_{11}=4 M e_{x x}$ is also a linear function of $y$ and generates in layer 1 a bending moment

$$
\mathscr{M}_{1}=\frac{1}{3} h_{1}^{3} M_{1} \frac{\alpha_{2}\left(L_{1}-L_{2}\right)}{\alpha_{1} L_{2}+\alpha_{2} L_{1}} \frac{\partial^{2} v}{\partial x^{2}} \text {. }
$$

The sign of $\mathscr{M}_{1}$ is chosen positive counterclockwise when acting on a solid lying to the left (see figure 3). Similarly in layer two the bending moment is

$$
\mathscr{M}_{2}=\frac{1}{3} h_{2}^{3} M_{2} \frac{\alpha_{1}\left(L_{2}-L_{1}\right)}{\alpha_{1} L_{2}+\alpha_{2} L_{1}} \frac{\partial^{2} v}{\partial x^{2}} .
$$

Let $h=\frac{1}{2}\left(h_{1}+h_{2}\right)$ be the average thickness of the layers. The total average bending moment per unit thickness is

$$
\mathscr{M}=\frac{1}{2 h}\left(\mathscr{M}_{1}+\mathscr{M}_{2}\right)=b \frac{\partial^{2} v}{\partial x^{2}},
$$

with the couple-stress coefficient

$$
b=\frac{4}{3} h^{2}\left(\alpha_{1}^{2} M_{1}-\alpha_{2}^{2} M_{1}\right) \frac{\left(L_{1}-L_{2}\right) \alpha_{1} \alpha_{2}}{\alpha_{1} L_{2}+\alpha_{2} L_{1}} .
$$


This expression is considerably simplified if one layer is much more rigid than the other. For example, if $M_{1} \gg M_{2}$ and $L_{1} \gg L_{2}$ we obtain

$$
b=\frac{1}{3} h_{1}^{2} M,
$$

where $M$ is the average elastic coefficient (7.1) for the equivalent continuum.

In the subsequent analysis we shall assume that $b>0$.

\section{Validity of the couple-stress approximation}

The existence of a skin effect which depends on the magnitude of the anisotropy was brought out in a recent paper (Biot $1966 a$ ). It was found that the stress will vary rapidly near the boundary of a layer through a skin thickness $\delta=\mathscr{L} /(2 \pi \sqrt{ }(2 m))$ where $\mathscr{L}$ represents the wavelength along the layers. Obviously the wavelength must be large enough so that the skin thickness is not smaller than the thickness $h_{1}$ or $h_{2}$ of the laminations. For a given wavelength this puts a limitation on the magnitude of the anisolropy since the latter is measured by $m$. For isotropic materials $m=1$ and $\delta \cong 0 \cdot 1 \mathscr{L}$. Hence in this case the wavelength must not be less than about ten times the lamination thickness. The condition also applies to the collective anisotropy of the laminated medium itself considered as an approximate continuum. However, in this case closer examination of the mechanics indicates that this will only affect the stress field in localized boundary regions without changing appreciably the overall behaviour of the system.

A special correction must also be applied for internal buckling due to interstitial flow in the case of large rigidity contrast between the two lamination materials. This effect was evaluated earlier (Biot 1964a, 1965 $d$ ) and will be discussed briefly in the next section.

\section{Extension to viscous, visconlastic aNd PLAstic media}

The author's principle of viscoelastic correspondence (Biot $1955,1963 c, 1965 a$ ) is applicable to a medium under initial stress. By this principle extensions of the previous results to viscous and viscoelastic media is trivial, and is obtained by substituting operators for the moduli in the equations of the elasticity theory.

Consider, for example, the laminated medium. For layer 1 the viscoelastio properties are expressed by the operators

$$
\left.\begin{array}{c}
\hat{L}_{1}=\int_{0}^{\infty} \frac{p}{p+r} L_{1}(r) \mathrm{d} r+L_{1}+L_{1}^{\prime} p, \\
\hat{M}_{1}=\int_{0}^{\infty} \frac{p}{p+r} M_{1}(r) \mathrm{d} r+M_{1}+M_{1}^{\prime} p,
\end{array}\right\}
$$

with the operator

$$
p=\mathrm{d} / \mathrm{d} t
$$

which represents a time derivative. The viscoelastic properties of layer 2 are represented by operators $\hat{L}_{2}$ and $\hat{M}_{2}$ given by expressions similar to $(\mathbf{8} \cdot 1)$. These expressions were derived by the author from irreversible thermodynamics (Biot I954, 
I $963 c, 1965 a)$. The collective elastic coefficients $L$ and $M$ of the equivalent continuum must be replaced by the operators

$$
\begin{gathered}
\hat{M}=\hat{M}_{1} \alpha_{1}+\hat{M}_{2} \alpha_{2}, \\
\hat{L}=1 /\left(\frac{\alpha_{1}}{\hat{L}_{1}}+\frac{\alpha_{2}}{\hat{L}_{2}}\right) .
\end{gathered}
$$

The couple-stress coefficient $(7 \cdot 10)$ becomes the operator

$$
\hat{b}=\frac{4}{3} h^{2}\left(\alpha_{1}^{2} \hat{M}_{1}-\alpha_{2}^{2} \hat{M}_{2}\right) \frac{\left(\hat{L}_{1}-\hat{L}_{2}\right) \alpha_{1} \alpha_{2}}{\alpha_{1} \hat{L}_{2}+\alpha_{2} \hat{L}_{1}} .
$$

It should be noted that the foregoing operators represent incremental properties. They relate theincremental deformations to increments of stresses and couplestresses superposed upon a state of initial stress. In other words the theory is applicable to material which are incrementally viscous or viscoelastic.

Modes of viscoelastic instability are obtained by solving the characteristic equation. The equation is formally the same as for the elastic case with corresponding operators substituted for the elastic coefficient. The time derivative $p$ now becomes a real positive quantity. Each positive value of $p$ which satisfies the characteristic equation yields a mode of viscoelastic instability whose amplitude is proportional to $\exp (p t)$.

In some cases incremental deformations may exhibit purely viscous behaviour. This corresponds to operators $\hat{M}_{1}=M_{1}^{\prime} p, \hat{M}_{2}=M_{2}^{\prime} p, \hat{L}_{1}=L_{1}^{\prime} p, \hat{L}_{2}=L_{2}^{\prime} p$. In such a case the average operators $(8 \cdot 3)$ and the couple-stress operator $(8 \cdot 4)$ may be written

$$
\left.\begin{array}{rl}
\hat{M} & =M^{\prime} p \\
\hat{L} & =L^{\prime} p \\
\hat{b} & =b^{\prime} p
\end{array}\right\}
$$

The coefficients $M^{\prime}, L^{\prime}$ and $b^{\prime}$ are viscosity coefficients similar to those of an anisotropic fluid with couple stresses. Their values are easily derived from equations $(8 \cdot 3)$ and $(8 \cdot 4)$.

Note that this is rigorously valid for a material which is at rest under the initial stress and exhibits purely viscous properties for incremental deformations. Actually the result constitutes a suitable approximation for a medium which is not rigorously at rest initially but is in a state of slow creep. The theory becomes thereby applicable to strongly nonlinear plastic materials and the viscosity coefficients become differential coefficients representing the local slopes of strain-rate curves plotted as a function of stress (Biot $196 \mathrm{r}, 1965 a$ ). For a wide range of problems the approximation is also applicable to a Newtonian or anisotropic fluid under initial stress. The validity of this approximation was discussed elsewhere (Biot $1965 a, e)$.

\section{Hydrodynamic instability due to viscosity stratification}

An exact theory of folding instability of a fluid with stratified Newtonian viscosity was developed earlier (Biot $1964 b, 1965 a$ ). This provides a rigorous treatment of folding instability for a medium which is not at rest under the initial stress but in a state of flow with a time-history of finite strain. 


\section{Comparison between fluid mechanics and viscoelasticity}

In this connexion reference should be made to a paper by Ramberg (I964) where the analysis of folding instability is alleged to be based on fluid dynamics. This statement by Ramberg is incorrect since he does not take into account the fact that the fluid is not at rest under the initial stress and that the folding is superposed on an initial state of flow. Actually Ramberg's treatment is equivalent to considering a medium initially at rest which is incrementally viscous and isotropic and is therefore a particular case of a viscoelastic medium with operators $\hat{L}=\hat{M}=\eta p$ ( $\eta=$ viscosity). It is not surprising therefore that Ramberg's results are in complete agreement with those derived earlier by the author under the same assumptions. However, the incrementally viscous model initially at rest is not theoretically correct for a Newtonian fluid but in practice it is generally applicable as an approximation to a viscous fluid. The range of validity of the approximation has been discussed in detail elsewhere (Biot $\left.1964 b, \mathrm{I}_{9} 65 a, c\right)$.

\section{Operational-variational principle}

Variational principles in operational form are immediately derived by viscoelastic correspondence (Biot $1955,{ }_{1965} a$ ) by the simple procedure of replacing the elastic coefficients by operators.

\section{Internal buckling of a laminated medium}

Equations for internal buckling of a medium with couple stresses are applicable to a laminated medium whose incremental properties are either elastic, viscous or viscoelastic. Results are formally the same for all cases by introducing the general operators $(8 \cdot 3)$ and $(8 \cdot 4)$. The same buckling configuration is obtained as shown in figure 4.

Consider, for example, the case of a laminated elastic medium where one layer is much more rigid than the other. The couple-stress coefficient in this case is given by expression (7.11). Since $M / L$ is large we may write approximately

$$
\left.\begin{array}{rl}
\frac{a}{m} & =\frac{\pi^{2}}{6} \frac{h_{1}^{2}}{H^{2}}, \\
a m & =\frac{2}{3} \pi^{2} \frac{h_{1}^{2}}{H^{2}} \frac{M^{2}}{L^{2}} .
\end{array}\right\}
$$

Because $a / m$ is small, expressions $(5 \cdot 15)$ and $(5 \cdot 16)$ are valid. By introducing the value (8.6) for $a / m$ the buckling wavelength $(5 \cdot 15)$ becomes

$$
\mathscr{L}=1 \cdot 90 \sqrt{ }\left(h_{1} H\right) .
$$

This same value is obtained for the dominant wavelength for a medium which incrementally is purely viscous and is represented by the operators (8.5). Hence, in first approximation, the folding is governed mainly by the geometry.

This remarkably general and simple result represented by equation (8.7) was already obtained earlier (Biot $1964 a, 1965 d$ ) in a less general analysis. 
For the elastic medium, equation $(5 \cdot 16)$ giving the buckling load $P$ may be written

$$
\frac{P}{4 M}=\frac{1}{4} \frac{L}{M}+1 \cdot 81 \frac{h_{1}}{H}
$$

A similar equation is obtained for the incrementally viscous medium by substituting for $L$ and $M$ the operators $L^{\prime} p$ and $M^{\prime} p$. We obtain

$$
\frac{P}{4 M^{\prime} p}=\frac{1}{4} \frac{L^{\prime}}{M^{\prime}}+1 \cdot 81 \frac{h_{1}}{H} \text {. }
$$

The amplitude of folding of the dominant wavelength grows proportionally to $\mathrm{e}^{p}$ under a given applied compressive load $P$. The rate of growth of the folding is represented by the value of the coefficient $p$ and determined by equation (8.9).

\section{Interstitial flow correction}

When the rigidity contrast becomes very large a correction must be introduced which takes into account the local flow of matter which occurs in the soft layers relative to the more rigid layers. We shall consider layer 2 as the soft layer. This 'interstitial flow' effect was analysed earlier (Biot $1964 a, 1965 d$ ) in a special case. However, the result is quite general and may be formulated as an apparent anisotropic compressibility, which amounts to replacing condition $(2 \cdot 4)$ for incompressibility by

$$
e_{x x}+e_{y y}=-\frac{1}{12} \frac{h_{2}^{3}}{\hat{L}_{2}} \frac{\partial^{2} t_{22}}{\partial x^{2}}
$$

This equation makes the strain dependent on the second gradient of the stress. Othcr equations remain unchanged. The effect of interstitial flow on the wavelength of internal buckling was evaluated approximately for the particular case

$$
\hat{L}_{1}=\hat{M}_{1}=\eta_{1} p, \quad \hat{L}_{2}=\hat{M}_{2}=\eta_{2} p
$$

(Biot $1964 a, 1965 d$ ). The dominant wavelength was found to be

$$
\mathscr{L}=1 \cdot 90 \sqrt{ }\left(h_{1} H\right)\left(1+3 \cdot 63 \frac{\eta_{1} \alpha_{2}^{3}}{\eta_{2} n}\right)^{\frac{1}{6}} .
$$

In this case $\eta_{1}$ and $\eta_{2}$ represent isotropic viscosity coefficients of the hard and soft layers respectively and $n$ is the total number of confined layers. The same correction factor applies for the buckling wavelength for a purely elastic material if we replace $\eta_{1} / \eta_{2}$ by the ratio $\mu_{1} / \mu_{2}$ of the elastic moduli of the two layers.

Note that the correction factor is extremely insensitive to the physical parameters because it depends only on the $\frac{1}{6}$ power. Moreover the correction is negligible if $3 \cdot 63 \eta_{1} \alpha_{2}^{3} /\left(\eta_{2} n\right)<1$.

\section{General formulation of geological Folding}

The theory of stability of multilayers with couple stresses outlined in $\S 6$ may be applied to the case where the layers themselves are constituted by laminated materials. Structures of this type are frequently encountered in geology. For example, figure 6 
shows the superposition of four horizontal layers, where layers 1, 2 and 3 are laminated media, while layer 4 is homogeneous and isotropic. The laminated layers $1,2,3$ are themselves constituted by a superposition of alternating hard and soft layers and each of them is approximated by an anisotropic continuum with couple stresses. The average effective initial compressive stress $P$ would also be different in each of the four layers.

Let us assume that the structure is subject to horizontal compression associated with a state of slow creep and a homogeneous strain. The rheological properties of the

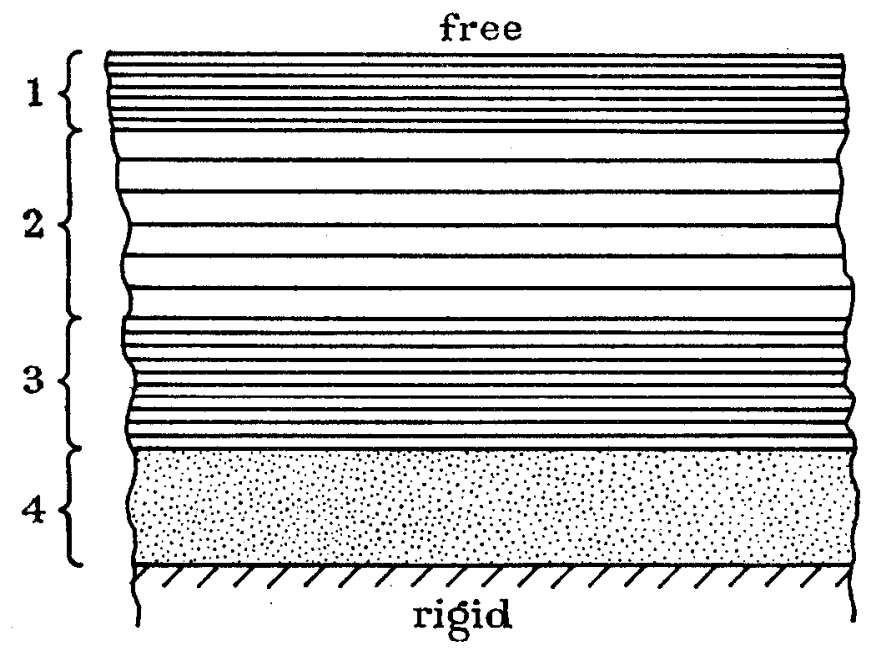

FIGURE 6. Example of a stratified geological structure analysed as a four-layer system. Layers 1,2 and 3 are considered to be laminated plates each behaving as a continuous anisotropic medium with couple stresses.

materials may correspond to nonlinear plastic behaviour. However, incremental deformations representing deviations from the initial state of homogeneous creep may be represented by viscous properties expressed by the operators (8.5).

The problem of deriving the time history of folding instability of the structure is solved by applying the general equations of $\S 6$. The effect of gravity and density contrast is included in these equations. Suitable operators are substituted for the elastic constants to represent the incremental viscous properties. The unknowns are the two displacements $U$ and $V$ at each interface or free surface. Note that the structure illustrated in figure 6 is analysed as a four-layer system, hence the interfaces referred to here are those between the composite laminated layers as a whole and not those within the components of the laminations. This means that there are only eight unknowns in the stability equations. They are the two displacements at the free top surface, and at the three interfaces between the component layers 2,3 and 4 . The displacements at the bottom interface of layer 4 vanish if we assume the structure to rest without friction on an 'incrementally rigid' medium. IIence the characteristic equation of the stability problem is an $8 \times 8$ determinant. Solutions of this equation determines the parameter $p$ as a function of the wavelength. Folds 
of amplitude proportional to $\mathrm{e}^{p t}$ are obtained and maximum values of $p$ determine the dominant wavelengths. By the same token the time history of folding may be evaluated starting with an initial deviation of the structure from perfect flatness and representing the deviation by a superposition of sinusoidal solutions and orthogonal characteristic solutions.

\section{Applichtion to typical geological structures}

Preliminary approximate and simplified solutions previously derived by the author in key problems lead to the prediction of the behaviour of the more complex structures which can be analysed by the more exact but more elaborate methods developed in the present paper.

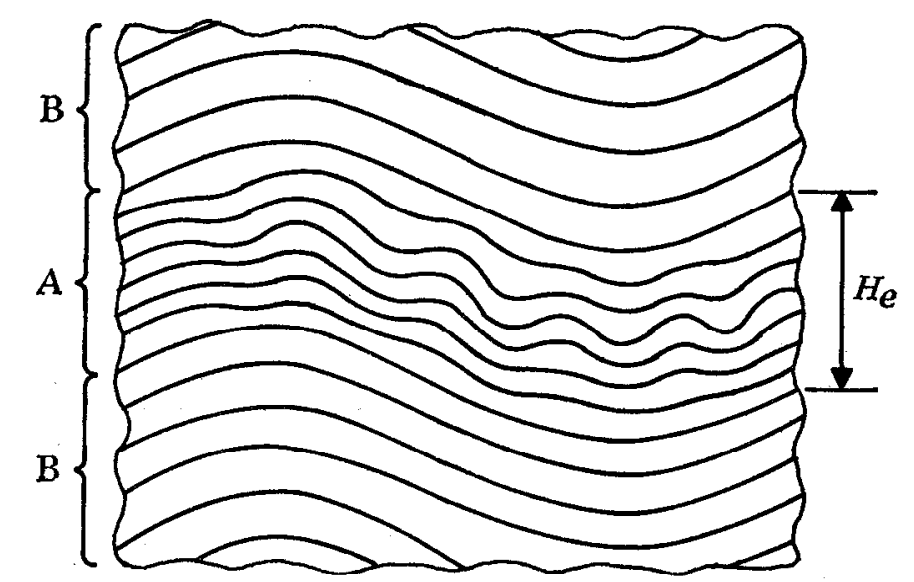

Figure 7. Folded structure showing the simultaneous appearance of two dominant wavelengths.

\section{Simultaneous appearance of different folding wavelengths}

Under conditions of confinement equation (8.7) is approximately valid and shows that the dominant wavelength depends primarily on two geometric parameters, the thickness, $h_{1}$, of the competent layers and the confinement distance, $H$. On the other hand the rate of growth of the folding is relatively insensitive to scale and depends more on material properties and geometric ratios. This may be seen by considering the value of $p$ obtained from equation (8.9). This value of $p$ determines the rate of folding and may be the same for two different dominant wavelengths as illustrated in figure 7. In region A the layers are thinner with an effective confinement distance $H_{e}$, corresponding approximately to the distanco between regions $\mathrm{B}$. With $h_{1}$ denoting the competent layer thickness in region $A$ the dominant wavelength in this region is about $\mathscr{L}=1.90 \sqrt{ }\left(h_{1} H_{e}\right)$. In the overall region A and B both the competent layer thickness and the confinement distance are large, resulting in a larger dominant wavelength superimposed on the smaller folds. As a rule the larger wavelengths will be superposed on the smaller folds but the reverse will not be true. 
Confined microstructures

Internal buckling of a thinly laminated structure of schistous or micaceous rock confined between thick competent layers will generate microfolds as illustrated in figure 8 . The dominant wavelength or buckling wavelength is given by the same formula (8.7), whether the medium is elastic or in a state of quasiviscous creep. Equation (8.7) has recently been applied (Potter I966) to micaceous deposits between competent sandstone layers. Results are in good agreement with field observations.

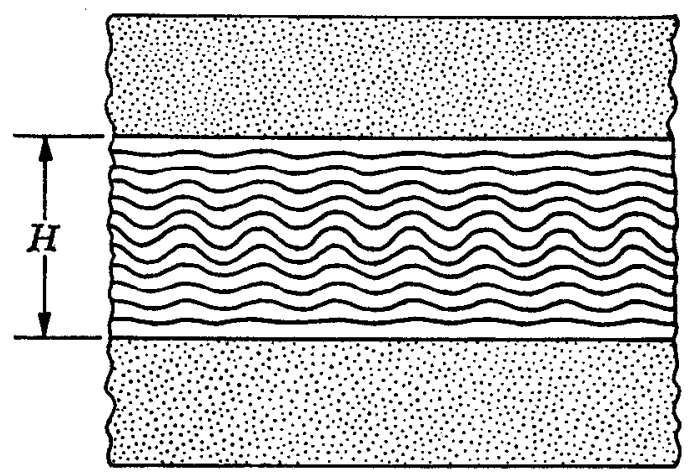

FiGURE 8. Folding in thinly laminated microstructure of micaceous or schistous rock confined between thick competent layers.

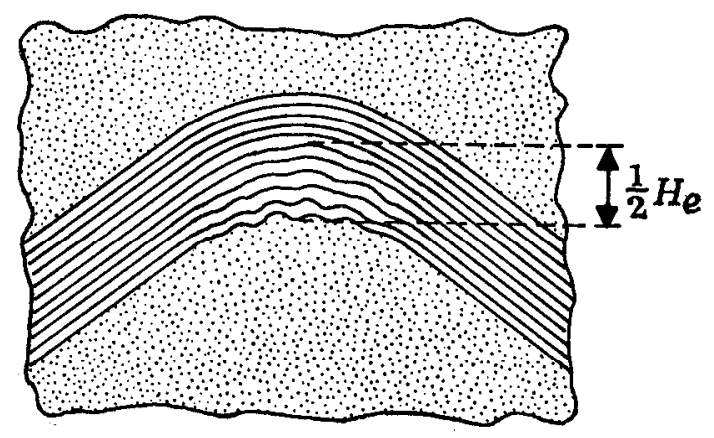

FiguRe 9. Secondary folding in the compressed region of a crest.

\section{Secondary folding}

The compression which causes the folding instability may itself be generated by localized stresses due to primary larger scale folding. As an example consider the crest region in a folded competent thinly laminated layer illustrated in figure 9. The compressed region may be considered as a laminated medium with an effective confinement distance. Secondary microfolding will appear in this region. The effective confinement distance $H_{e}$ may be evaluated by applying the general theory in the present paper. As a preliminary approximation we may assume that $H_{e}$ is about equal to the total thickness of the laminated layer. Microstructures of this type in 
sedimentary rock are quite common. It has also been observed in layered rubber models (Ramberg 1964).

\section{Similar folding of the first and second kind: transition to internal buckling}

A model of particular interest is illustrated in figure 10, where a multilayered structure A lies on top of a thick soft layer B, and the whole system rests on a rigid foundation. The folding of this system, including the effect of gravity, may be analysed by applying the general equations of $\S 6$, for a large category of materials, incrementally elastic, viscous or viscoelastic. The four unknowns are the two displacements at the top and bottom of region $A$ and the characteristic equation is a $4 \times 4$ determinant. Particular cases of this model have been analysed previously for an infinitely thick region B (Biot 196I) and for the case of pure gravity instability (Biot \& Odé 1965 ; Biot $1966 \mathrm{~b}$ ). The latter treatment included the effect of variable thickness and compaction of the overburden with special reference to the formation of salt structures.

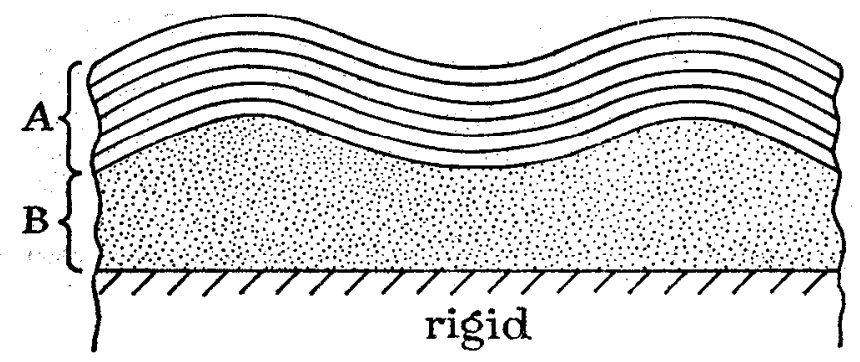

Figdre 10. Folding and gravity instability of a stratified overburden separated from a rigid base by a soft layer.

A previous approximate analysis of folding of the analogous case of a multilayered structure embedded in a soft medium (Biot $1965 b$ ) permits certain conclusions regarding the general behaviour of the structure shown in figure 10. Under certain conditions when layer $B$ is very soft the folding wavelength will be large, resulting in what the author has referred to as similar folding of the first kind. In this case the multilayered overburden A exhibits almost pure bending behaving as a single anisotropic layer. For increasing stiffness of layer B the wavelength decreases. Below a cross-over wavelength depending on the collective anisotropy of the overburden the folding is predominantly a shear instability which we have referred to as similar folding of the second kind. For further increase of rigidity of region B the overburden $A$ becomes more and more confined at the bottom and internal buckling will begin to show up. This is characterized by a variation in folding amplitude across the thickness of the multilayered overburden.

\section{Plastic flow and nonlinear effects}

The foregoing linearized analysis will be approximately valid for folding with moderate slopes. In this initial phase as shown in a previous analysis for the single layer (Biot $1964 b, 1965 a, c)$ due to the compensating effects of overall compressive shortening and thickening of the layers, the wavelength of the folding tends to 
remain constant. Beyond a certain amplitude the bending stress at the crest produces a local yielding (Biot $196 \mathrm{I}$ ). In this second phase the crests behave as sharp hinges and the wavelength decreases in accordance with further compressive shortening. The analysis shows that the onset of the plastic phase is delayed to larger folding amplitudes when the compressive stress decreases.

An entirely similar behaviour may be exhibited by a multilayered structure when the second phase of similar folding is characterized by sharp folds with large slopes which participate in the overall compressive shortening of the whole structure.

\section{REFERENOES}

Biot, M. A. 1939 Non-linear theory of elasticity and the linearized case for a body under initial stress. Phil. Mag. 27, 468-489.

Biot, M. A. I954. Theory of stress-strain relations in anisotropic viscoelasticity and relaxation phenomena. J. Appl. Phys. 25, 1385-1391.

Biot, M. A. 1955 Variational and Lagrangian methods in viscoelasticity. Proc. IUTAM colloquium on deformation and flow in solids (Madrid 1955), pp. 251-263. Berlin : Springer.

Biot, M. A. 1959 The influence of gravity on the folding of a layered viscoelastic medium under compression. J. Franklin Inst. 267, 211-228.

Biot, M.A. I96I Theory of folding of stratified viscoelastic media and its implications in tectonics and orogenesis. Bull. Geol. Soc. Am. 72, 1595-1620.

Biot, M. A. 1963a Internal buckling under initial stress in finite elasticity. Proc. Roy. Soc. A 273, 306-329.

Biot, M. A. ${ }_{1963} b$ Theory of stability of multilayered continua in finite anisotropic elasticity. J. Franklin Inst. 276, 128-153.

Biot, M. A. I963 $c$ Stability of multilayered continua including the effect of gravity and viscoelasticity. J. Franklin Inst. 276, 231-252.

Biot, M. A. r964a Theory of internal buckling of a confined multilayered structure. Bull. Geol. Soc. Am. 75, 563-568.

Biot, M. A. $1964 b$ Theory of viscous buckling of multilayered fluids undergoing finite strain. Physics Fluids 7, 855-859.

Biot, M. A. I965a Mechanics of incremental deformations. New York: John Wiley and Sons, Inc. $504 \mathrm{pp}$.

Biot, M. A. 1965 $b$ Theory of similar folding of the first and second kind. Bull. Geol. Soc. Am. 76, 251-258.

Biot, M. A. I965c Theory of viscous buckling and gravity instability of multilayers with large deformations. Bull. Geol Soc. Am. 76, 371-378.

Biot, M. A. I965d Further development of the theory of internal buckling of multilayers. Bull. Geol. Soc. Am. 75, 833-840.

Biot, M. A. I965e Internal instability of anisotropic viscous and viscoelastic media under initial stress. $J$. Franklin Inst. 279, 65-82.

Biot, M. A. r966a Fundamental skin effect in anisotropic solid mechanics. International Journal of Solids and Structures 2, 645-663.

Biot, M. A. 1966b Three-dimensional gravity instability derived from two-dimensional solutions. Geophysics 31, 153-166.

Biot, M. A. \& Odé, H. 1965 Theory of gravity instability with variable overburden and compaction. Geophysics 30, 213-227.

Cauchy, A. L. I85 I Note sur l'équilibre et les mouvements vibratoires des corps solides. Comptes-Rendus 32, 323-326.

Cosserat, E. \& Cosserat, F. 1909 Théorie des corps déformables. Paris: A. Hermann et Fils.

Potter, J. F. 1966 Deformed micaceous deposits in the Downtonian of the Llandeilo region, South Wales. Proc. Geol. Ass., Lond. 77 (to be published).

Ramberg, H. I964 Selective buckling of composite layers with contrasted rheological properties, a theory for simultaneous formation of several orders of folds. Tectonophysics 1, 307-341. 